\title{
Real-Time Holding Control for High-Frequency Transit with Dynamics
}

\author{
G.E. Sánchez-Martínez ${ }^{\mathrm{a}, *}$, H.N. Koutsopoulos ${ }^{\mathrm{a}, \mathrm{b}}$, N.H.M. Wilson ${ }^{\mathrm{a}}$ \\ ${ }^{a}$ Massachusetts Institute of Technology, Room 1-235, 77 Massachusetts Ave., Cambridge, \\ MA 02139 \\ ${ }^{b}$ Northeastern University, 360 Huntington Ave., Boston, MA 02115
}

\begin{abstract}
Operations control is an important means of improving service quality for highfrequency transit. Past research on real-time control has focused on developing and evaluating the effectiveness of different control strategies, largely relying on running times and demand which are assumed to be static. We formulate a mathematical model for holding control optimization that reflects dynamic running times and demand. The model can be used to produce a plan of holding times that accounts not only for the current state of the system, but also for expected changes in running times and demand. We evaluate the effectiveness of the model within a simulation environment. The results show that control based on dynamic inputs outperforms its static equivalent in high demand cases where passengers can be left behind at stops, and to a lesser extent in low to moderate demand cases with time-varying running times.
\end{abstract}

Keywords: transit operations, real-time control, holding, high-frequency transit, dynamic control

\section{Introduction}

Operations control is an important means of improving service quality in high-frequency public transport systems. It is based on continuous monitoring of the system and supply-side interventions with the aim of providing the best possible service to passengers with the available resources. Holding, the most commonly employed intervention, consists of intentionally delaying a vehicle, possibly at the expense of extending trip times for passengers on board, in order to reduce the waiting time of passengers who will board downstream. Previous research has established that holding is the single most effective type of intervention, and a number of methods to determine holding policies have

\footnotetext{
*corresponding author; Telephone number: +1.617.258.0699

Email addresses: gsanmar@mit.edu (G.E. Sánchez-Martínez), h.koutsopoulos@neu.edu (H.N. Koutsopoulos), nhmw@mit.edu (N.H.M. Wilson)
} 
been proposed and evaluated, ranging from simple heuristics to sophisticated model-based optimization. (Eberlein et al., 2001)

Past research has focused on evaluating the effectiveness of different control strategies, largely based on static assumptions with respect to both running time and demand. Within the family of real-time control strategies based on future system state prediction models, none to date have modeled running times and demand dynamically. Holding strategies based on static running times and demand respond to disruptions in the initial system state. For example, if a vehicle has been delayed, holding can help prevent bunching from occurring. However, these strategies cannot anticipate systematic changes in the operating environment and preemptively consider those changes in the production of a control plan. A strategy that models running times and demand dynamically can do this. For example, such a strategy may be able to anticipate how additional demand leading into the rush hour may cause disruptions, and therefore produce a control plan that recognizes that such disruptions may develop.

This has significant implications in the realm of real-time information and operations control. By being able to react not only to what is already known in the initial system state but also to what can be anticipated based on historical experience, the model proposed in this research could potentially improve performance beyond that achieved with previously developed control strategies. Modeling demand dynamically may lead to more accurate predictions of future vehicle loads, which could help avoid holding full vehicles, a control action that would extend trip times for passengers on board without shortening waiting times for passengers at downstream stops.

Future running times and demand could be estimated based on real-time information of current conditions in addition to historical or typical conditions. For instance, an unusually high demand observed in some part of the network might be used to infer additional demand in another part of the network due to transfers. Or an accident might be reported at some intersection, allowing an operator to anticipate traffic delays along a corridor before the congestion develops. The aim of this research is to develop and test a model that can be used in these dynamic contexts, focusing on cases where information about dynamics is known in advance.

The remainder of this paper is organized as follows: Section 2 reviews the literature, Section 3 presents the framework and mathematical model, Section 4 discusses the implementation of this model within a simulation model for evaluation purposes, Section 5 presents and discusses the results from the application of the control model, and Section 6 draws conclusions.

\section{Literature Review}

Early research on the holding problem did not consider the availability of real-time information (Osuna and Newell, 1972, Barnett, 1974, Newell, 1974, Turnquist and Blume, 1980, and Abkowitz and Lepofsky, 1990). Since then researchers have proposed a number of control strategies with control actions based on both typical system characteristics (e.g. running times and demand) 
and real-time information (e.g. vehicle locations). Holding strategies differ in objectives, underlying models and solution methods, and information utilized. Common objectives include schedule adherence (Adamski and Turnau, 1998), headway adherence (Rossetti and Turitto, 1998), headway regularity (Daganzo, 2009, Bartholdi and Eisenstein, 2012, and Berrebi et al., 2015), and minimization of user costs such as waiting and in-vehicle times (Eberlein et al., 2001, Delgado et al., 2009, Delgado et al., 2012, and Sáez et al., 2012). Schedule adherence is a suitable objective for long headway service, while headway regularity is a suitable objective for short headway service. Strategies based on user cost minimization employ mathematical programming or other optimization methods and a variety of information including vehicle locations, loads, and passenger arrival rates, sometimes in a rolling horizon formulation. In some cases holding is combined with other control strategies such as short-turning (Shen and Wilson, 2001), boarding limits (Delgado et al., 2009 and Delgado et al., 2012), and signal priority (Chandrasekar et al., 2002).

Control strategies are typically evaluated through simulation. Performance is measured in terms of passenger waiting times and trip times (expected values and variability), often complemented with measures of headway regularity, loads, etc. Researchers have been effective at using this evaluation framework to demonstrate how the performance of transit systems can improve with enhanced availability and utilization of information, as well as innovative prediction and control models.

Previous research has not addressed the value of modeling system characteristics dynamically. While many of the recently proposed strategies utilize real-time information, very few are able to consider predictions of future system states involving dynamic running times and demand. The few with that (limited) ability have been tested in simulation environments having timeindependent running times and demand (Dessouky et al., 2003, Daganzo, 2009, and Sáez et al., 2012). In other cases, the simulation environment features timedependent running times and demand, but the strategies used cannot model the dynamics and instead assume typical period-level constants.

Ibarra-Rojas et al. (2015) systematically review the literature on planning, operations, and control of transit systems. The remainder of this section summarizes prior works of particular relevance to this research.

Adamski and Turnau (1998) develop a set of holding control strategies based on control theory. The aim is primarily punctuality, but the authors suggest a variation that adapts the punctuality control procedure to achieve headway regularity.

Rossetti and Turitto (1998) develop a holding strategy based on a dynamic threshold. The threshold used to determine the desired preceding headway, and therefore holding time, of a vehicle is chosen from a range of thresholds including the scheduled headway, with the aim of reducing holding time, and hence invehicle time added by holding. The term dynamic refers to the threshold and not to the running time and demand information.

Eberlein et al. (2001) formulate the holding problem as a deterministic quadratic program in a rolling horizon scheme, allowing real-time information 
to be taken into account. Their model includes the effect of dwell time on vehicle delay and headways, and the optimization objective is to minimize total passenger waiting times. Running times between stations and passenger arrival rates are assumed constant over the rolling horizon. The formulation includes a constraint that prevents late vehicles from being held. The resulting program is non-convex quadratic. The researchers find that holding policies are mainly sensitive to vehicle headway patterns and much less sensitive to passenger demand patterns, and that the impact of holding a vehicle on the trajectories of vehicles upstream diminishes quickly.

Shen and Wilson (2001) formulate a mixed integer program for holding, short-turning, and expressing trains on an urban rail system in the case of minor disruptions. Passenger demand and running times between stations are treated as constants.

Chandrasekar et al. (2002) test the strategy of regulating bus spacing by simultaneously providing signal priority to the leading vehicle and holding the trailing vehicle when the space between the vehicles shortens. Since the control strategy is reacting to deviations from the target vehicle spacing, it is not able to produce control policies based on dynamic running times and demand.

Zhao et al. (2003) present a distributed control approach in which vehicles and stops act as agents that communicate in real-time to coordinate departure times of vehicles from stops. They analyze the performance of their strategy and other strategies using simulation under a variety of conditions (including long headway scheduled service), including random bursts of passenger arrivals. Although they assess the performance of the model under dynamic conditions, their strategy does not consider expected dynamics.

Dessouky et al. (2003) compare control strategies that depend on communication, tracking, and passenger counting technologies and those using only local information. The application of interest is schedule coordination at a terminal. Among the strategies considered, some make use of predicted arrival times at the terminal and loads, which makes it possible to account for dynamic running times and demand. However, the researchers do not focus on the value of capturing predicted dynamics from the operations control perspective. Their simulation model uses time-independent running time distributions and mean passenger arrival rate.

Sun and Hickman (2008) formulate a convex quadratic program with linear constraints for optimizing holding times of vehicles at multiple stations. They assume a constant passenger arrival rate and vehicle travel time between adjacent stops.

Puong and Wilson (2008) develop a real-time disruption response model for rail transit focusing on holding. The model is a non-linear mixed integer program and captures passengers left behind at stations. The researchers assume constant passenger arrival rates and dwell times.

Delgado et al. (2009) formulate a non-convex quadratic program with linear constraints for holding and boarding limits. The model captures vehicle capacity constraints, and can be used in a rolling horizon optimization application. Passenger arrival rates per stop and travel times between stops are assumed 
deterministic, known, and constant over the optimization horizon.

Daganzo (2009) develops a holding strategy in which vehicles are analyzed in pairs. The following vehicle of a vehicle pair is delayed by holding when the headway shortens, and instructed to speed up (perhaps through denied boardings) when the headway lengthens. The authors state that the model can be extended to time-dependent demand and running times by using runspecific parameters and making adjustments to average inter-stop running times. However, they do not test this, and the method, which looks only at vehicle pairs, is not able to capture dynamics of demand or running times happening farther in the future.

Yu and Yang (2009) propose a two-step holding strategy. In the first step, a support vector machine is used to predict whether or not a vehicle will depart early from the next stop if it is held at the current stop, possibly considering running time dynamics. Holding is only considered if a vehicle is early now and is also predicted to be early at its next stop. In the second step, a genetic algorithm is used to minimize a combination of waiting cost and in-vehicle cost. Passenger arrival rates are modeled as time-independent, and the dynamics of running time predictions are limited to the next stop. The simulation experiment shows that running times predicted by the support vector machine are more accurate than mean running times, and that their optimization-based strategy reduces passenger cost more than a schedule-based strategy.

Xuan et al. (2011) develop a holding control strategy based on a virtual schedule. A one parameter version of the method can be optimized in closed form and is shown to be near-optimal and to outperform other holding strategies. Mean running times between stations are modeled as time-independent constants and the passenger arrival process is assumed stationary.

Daganzo and Pilachowski (2011) develop a holding control model based on control theory. Headways are adjusted through holding considering the preceding and following headways. In their continuum idealization, the passenger arrival process is spatially homogeneous and time-independent. Vehicles are assumed to run at a fixed average speed between stops.

Cats et al. (2011) compare the effectiveness of holding based on schedule adherence, target headway, and even headway strategies. They find that the even headway strategy leads to the best performance in terms of headway regularity, trip time savings, and schedule adherence at a relief point. The strategies they consider are local and myopic (i.e. they do not involve prediction or optimization). Hence, they do not yield policies sensitive to time-dependent running times or demand.

Bartholdi and Eisenstein (2012) develop a strategy that holds vehicles based on their following headway, and show that it leads to even headways. The strategy can react to perturbations but does not take into account predicted future changes in running times or demand.

Delgado et al. (2012) formulate a non-linear model for optimization of holding times and metering of boardings of all vehicles of a transit line at all stops, taking into account vehicle capacity constraints. The model, which our research builds upon, can be used in a rolling horizon framework. The authors show its 
effectiveness in simulation experiments. The model assumes time-independent passenger arrival rates and running times between stops.

Sáez et al. (2012) propose a control strategy that models demand stochastically and involves a discrete-time event-based predictive model. The strategy is applied in a rolling horizon framework and can suggest holding and expressing control actions. A genetic algorithm is used to identify control actions in reasonable computation times. The authors test the strategy in simulations with constant vehicle speed and Poisson demand having constant arrival rates by origin-destination pair. Muñoz et al. (2013) compare the holding strategy of this paper to that of Delgado et al. (2012).

Chen et al. (2013) investigate the strategy of holding a group of buses at one or more control points, considering boardings while holding. Their formulation uses constant deterministic running times between stops and passenger arrival rates.

Berrebi et al. (2015) formulate a stochastic model for the optimization of holding times with the objective of minimizing mean passenger waiting times. They then propose an approximate policy based on this model, which they find closely matches the optimal policy. The authors test their strategy in simulations with stationary stochastic running times and demand, as well as in one case with dynamic demand, and find improvements in performance compared to simpler headway-based holding strategies. Their model and holding policy do not explicitly capture dynamically evolving running times and demand, nor do they capture capacity constraints.

Hernández et al. (2015) analyze holding control strategies applied in a corridor with multiple bus services. They compare cases in which a central operator seeks to maximize service quality globally to those in which different operators control their lines independently. Their simulations, of a system with fixed running times and passenger arrival rates, show that centralized control leads to a reduction in user costs.

This paper extends the literature of real-time control for high-frequency transit services by presenting a model that explicitly incorporates the dynamic nature of running times and demand. The following sections present the proposed model framework and an evaluation of its performance.

\section{Framework and Formulation}

In this section we present the framework and formulation of a deterministic rolling-horizon optimization model of a high-frequency transit system. Our model extends the work of Delgado et al. (2012). It takes as inputs dynamic running time and demand functions, current system state (e.g. vehicle positions, load estimates, and estimated number of passengers currently waiting at stops), and a set of planned holding times for each vehicle at each stop. It outputs predicted future states (e.g. departure times and loads) through the end of the optimization horizon.

Vehicle movement is modeled with time-varying running times. Dynamic running times could represent mean running times between stops as a function 


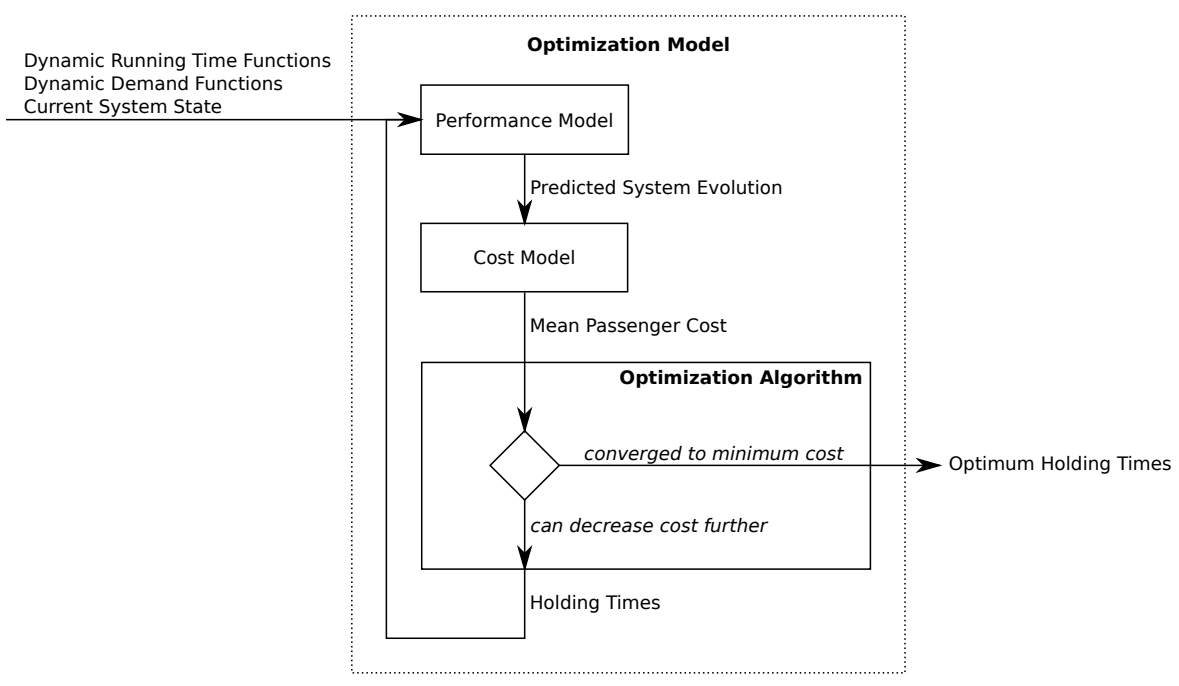

Figure 1: Optimization Framework

of time of day, which could be obtained using automated vehicle location technology. Demand is modeled with time-varying mean arrival rates at the origindestination pair level, allowing the use of time-dependent origin-destination matrices. Recent research in the use of automatically collected data to infer travel patterns enables estimating dynamic origin-destination matrices (Gordon et al., 2013). Matrix estimation methods such as iterative proportional fitting may be used when only boarding and alighting data are available (McCord et al., 2010).

The model is used to optimize holding times at any stop where control can be applied. Figure 1 illustrates how the different model components interact. The framework consists of an optimization model with three components: a performance model, a cost model, and an optimization algorithm. The performance model uses dynamic running time and demand functions, the current system state, and candidate holding times to predict (with some error) how the system will evolve. The prediction, which includes vehicle arrival and departure times, boardings and alightings, passengers left behind, and loads for all vehicles at all stops, is passed to the cost model, which gives a scalar mean cost per passenger reflecting waiting times and in-vehicle delay due to holding. The optimization algorithm considers the costs of multiple candidate solutions to minimize cost and suggest optimum holding times. The optimization process and the performance and cost models are described in Sections 3.2, 3.3, and 3.4, respectively. A list of mathematical notation can be found in Appendix A.

All of this takes place in the context of a rolling prediction horizon, which is defined (as in Delgado et al., 2012) to cover the departure of each vehicle from every stop once, starting with the next stop to be visited and finishing with the previously visited stop, to complete a cycle. This is illustrated in Figure 2 for two vehicles. Since the horizon boundary is defined spatially (i.e. a fixed 


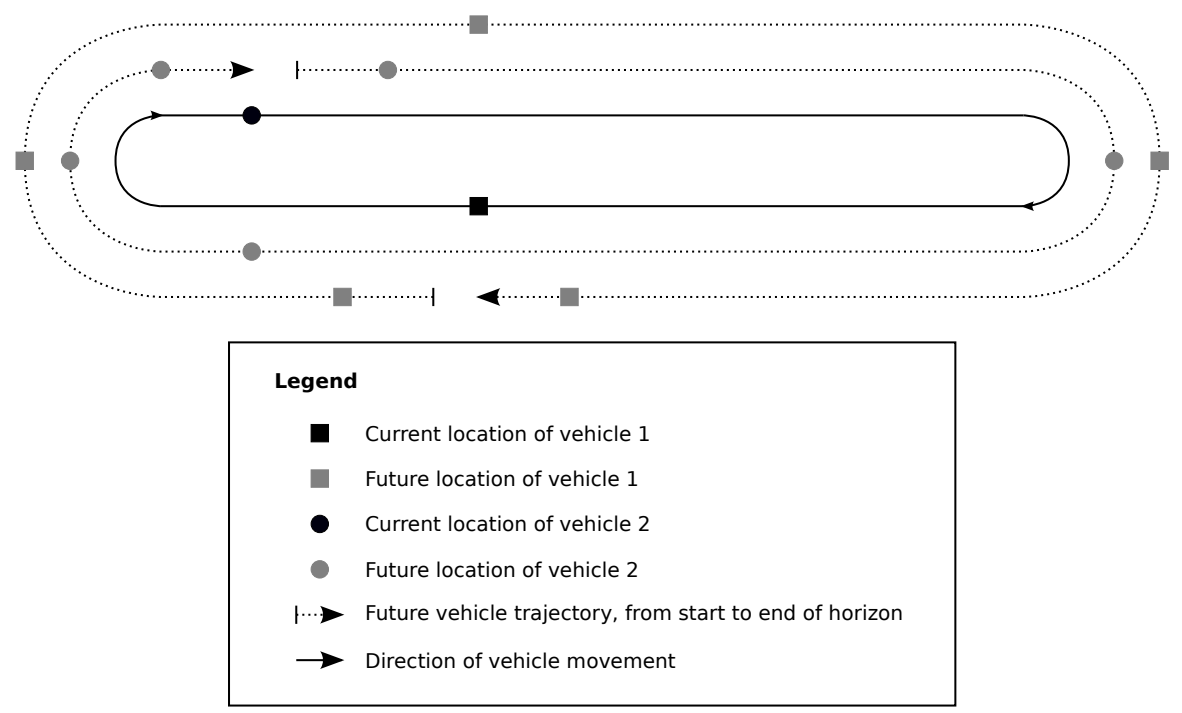

Figure 2: Rolling Prediction Horizon

number of stops), each vehicle's last stop visit in the horizon may happen at a different time.

The performance model can be used to make a prediction of how the system will evolve outside the optimization context. This is useful to estimate future arrival and departure times at stops considering time-dependent running times and demand.

\subsection{Assumptions}

The following assumptions are made:

1. The model is deterministic. Stochasticity, which is the very phenomenon that leads to the need for operations control, is neglected. This is done in the interest of tractability. This assumption is stronger when the model is applied to systems operating in a shared right of way, since traffic and signalized intersections can be major sources of stochasticity. Neglecting stochasticity leads to prediction errors that can accumulate over time. We hypothesize that the current state and the early parts of the model horizon, which should have less error than parts later in the horizon, are the most relevant for control decisions.

2. Passenger demand is modeled with continuous variables. This allows a simplified model of dwell times and avoids modeling discrete events such as the boarding of an individual passenger.

3. Vehicles stop at every stop. This assumption avoids decision variables concerning the conditions under which a vehicle would skip a stop. This assumption is accurate for rail systems, but may have an effect in bus 
systems if many stops are skipped and a significant portion of running times is deceleration and acceleration times at stops.

4. Vehicle order is preserved over the prediction horizon. Allowing overtaking would be of interest for bus systems in which overtaking happens frequently or when the insertion and removal of vehicles throughout the day is to be modeled, but the relaxation of overtaking constraints would require structural changes to the model that are outside the scope of this research.

5. Vehicles have a known, fixed capacity. The effective capacity of vehicles in real systems depends on the willingness of passengers to ride uncomfortably, which can vary by time of day, trip purpose, sociodemographic characteristics, and the expected waiting time for the following vehicle, as well as its uncertainty.

These assumptions concern the model and, except the last, do not necessarily apply to the simulation used to evaluate the effectiveness of the proposed control strategy.

\subsection{Holding Time Optimization}

The performance and cost models can be used to predict vehicle trajectories and passenger costs given a particular set of holding times for each vehicle and stop in a system with $n_{V}$ vehicles and $n_{S}$ stops. Treating them as a mathematical function $f: \mathbb{R}^{n_{V} n_{S}} \mapsto \mathbb{R}$ mapping a set of holding times $h_{v, s}$ to a scalar cost, they can also be used to obtain a set of optimal holding times in an optimization context. The objective is to minimize mean cost per passenger over the prediction horizon, which combines waiting time and in-vehicle delay, subject to constraints on holding times at stops:

$$
\begin{aligned}
\underset{h_{v, s} \forall v \in V \forall s \in S}{\operatorname{minimize}} & \frac{W_{V}+\theta_{S} W_{S}}{P} \\
\text { subject to } & \text { vehicle movement constraints } \\
& \text { passenger activity constraints } \\
& 0 \leq h_{v, s} \leq h_{s}^{\max } \quad \forall v \in V \quad \forall s \in S
\end{aligned}
$$

where $W_{V}$ and $W_{S}$ are the total in-vehicle delay and waiting time for all passengers in the prediction horizon, $P$ is the total number of passengers, and $\theta_{S}$ determines the relative disutility of waiting at a bus stop with respect to delay inside a vehicle. The objective function excludes time in motion because the small changes in running times that could result from holding, distributed throughout the entire trip, are less onerous than the more strongly perceived waiting time and in-vehicle delays due to holding.

Constraints (2) and (3) are handled by the performance model presented in Section 3.3. Constraint (4) states that holding times must be non-negative and may not exceed the maximum allowed by policy. For example, a maximum holding time of two minutes might be set at stops where holding is allowed, while longer maximums can be set at terminals having designated space for vehicles 
to hold without interfering with other vehicles in operation. The upper bound can be made very large, effectively removing this constraint, and it can also be set to zero, effectively preventing holding at a particular location.

Section 3.4 presents the mathematical formulation of the cost model. The objective function is not globally convex, so there may be multiple local optima. In this research, the term optimum refers to the lowest cost solution found by the optimization algorithm.

The Apache Commons Math implementation of the BOBYQA optimization algorithm by Powell (2009) is used. BOBYQA minimizes non-linear functions subject to bound constraints such as (4) by iterative quadratic approximation of a trust region, without requiring explicit derivatives. Quadratic approximations are derived from interpolation points obtained by evaluating the function being minimized. In this research, the optimization algorithm calls the performance and cost models to evaluate the objective function for each candidate set of holding times.

\subsection{Performance Model}

The performance model makes a deterministic prediction of how the system will evolve over the rolling prediction horizon, based on dynamic mean running times and passenger arrival rates. The prediction includes vehicle arrival and departure times, running times between stops, boardings and alightings, dwell times, and number of passengers left behind due to capacity constraints. The prediction of the future states of the system is used by the cost model to calculate the cost associated with a candidate set of holding times.

Stops are labeled in order from 1 to $n_{S}$ and vehicles from 1 to $n_{V}$, with stop $n_{S}$ and vehicle 1 being the furthest downstream stop and vehicle, respectively. The formulation uses rolling addition and subtraction for vehicle and stop indexes. For example, if $v=1, v-1=n_{V}$, and if $s=n_{S}, s+1=1$. Greek letters are used to represent passenger activity. Primed variables (e.g. $\left.d^{\prime}\right)$ represent known quantities observable before the current time $t_{0}$. Barred variables (e.g. $\bar{d})$ represent constrained quantities.

The arrival time $a_{v, s}$ of a vehicle $v$ at its first stop in the rolling horizon (stop $s=e_{v}+1$ ) is obtained by adding its running time to the departure time from the previously visited stop. This departure time $d_{v, e_{v}}^{\prime}$ is a constant that was observed before $t_{0}$. Throughout this formulation, dynamic running times take the form $r_{s}(t)$, where $s$ is the departure stop and $t$ is the departure time of a vehicle next visiting stop $s+1$. The unconstrained running time is determined by evaluating the dynamic running time function $r_{e_{v}}(t)$ of the vehicle's initial stop ( $\operatorname{stop} e_{v}$ ) at the vehicle's departure time.

$$
a_{v, s}=d_{v, e_{v}}^{\prime}+r_{e_{v}}\left(d_{v, e_{v}}^{\prime}\right) \quad \forall v \in V \quad s=e_{v}+1
$$

The model requires preservation of vehicle order throughout the optimization horizon. Extra running time $\bar{r}_{v}^{\prime}$ can be added to prevent a vehicle from arriving at a stop before the preceding vehicle.

$$
\bar{r}_{v}^{\prime}=\max \left(0, a_{v-1, s}^{\prime}-a_{v, s}\right) \quad \forall v \in V \quad s=e_{v}+1
$$


Typical running time functions $r_{s}(t)$ satisfy $\frac{\mathrm{d} r_{s}}{\mathrm{~d} t}(t) \geq-1$, preventing a vehicle from arriving earlier at the next stop by delaying its departure from its current stop. In this case, the running time function guarantees that $a_{v, s} \geq a_{v-1, s}^{\prime}$, and $\bar{r}_{v}^{\prime}=0$. However, the link overtaking constraint makes the model robust to running time functions that do not satisfy this property, which could happen when they are estimated from historical running time data. The preceding vehicle's arrival time is known because it occurs before $t_{0}$.

The (possibly) constrained arrival time $\bar{a}_{v, s}$ at a vehicle's first stop is the unconstrained arrival time plus any extra running time required to prevent overtaking.

$$
\bar{a}_{v, s}=a_{v, s}+\bar{r}_{v}^{\prime} \quad \forall v \in V \quad s=e_{v}+1
$$

These terms collectively represent an estimate of a vehicle's arrival time at its first stop in the optimization horizon. Unconstrained arrival times at subsequent stops are obtained from the addition of running time to the departure time from the previous stop, $d_{v, s-1}$. Running times $r_{s}(t)$ are dynamic functions.

$$
a_{v, s}=d_{v, s-1}+r_{s-1}\left(d_{v, s-1}\right) \quad \forall v \in V \quad \forall s \in\left[e_{v}+2, e_{v}\right]
$$

Extra running time may have to be added to prevent overtaking, as before, if running time functions do not satisfy $\frac{\mathrm{d} r_{s}}{\mathrm{~d} t}(t) \geq-1$. This applies starting at the first stop that a vehicle visits following a visit by the preceding vehicle at the same stop in the optimization horizon. An exception is made for the first vehicle (i.e. the one furthest downstream) in the special case that all vehicles are between the same two stops at the beginning of the optimization horizon; otherwise the model could be infeasible.

$$
\bar{r}_{v, s}= \begin{cases}\max \left(0, \bar{a}_{v-1, s+1}-a_{v, s+1}\right) & \forall v \in V \quad \forall s \in\left[e_{v-1}, e_{v}-1\right] \\ & \text { unless } v=1 \text { and } e_{1}=e_{n_{V}} \\ 0 & \text { otherwise }\end{cases}
$$

The (possibly) constrained arrival time is the unconstrained arrival time plus any extra running time required to prevent overtaking.

$$
\bar{a}_{v, s}=a_{v, s}+\bar{r}_{v, s-1} \quad \forall v \in V \quad \forall s \in\left[e_{v}+2, e_{v}\right]
$$

Unconstrained departure times $d_{v, s}$ are obtained by adding dwell time $\delta_{v, s}$ and holding time $h_{v, s}$ to arrival times.

$$
d_{v, s}=\bar{a}_{v, s}+\delta_{v, s}+h_{v, s} \quad \forall v \in V \quad \forall s \in S
$$

In order to prevent overtaking, it is necessary to delay a vehicle's departure by $\bar{h}_{v, s}$ when the preceding vehicle has not yet departed the same stop. As with arrival times, the overtaking constraint starts to apply at the first stop that a vehicle visits following a previous visit in the optimization horizon, and an exception is made for the first vehicle in the special case that all vehicles are between the same two stops at the beginning of the optimization horizon.

$$
\bar{h}_{v, s}= \begin{cases}\max \left(0, \bar{d}_{v-1, s}-d_{v, s}\right) & \forall v \in V \quad \forall s \in\left[e_{v-1}+1, e_{v}\right] \\ & \text { unless } v=1 \text { and } e_{1}=e_{n_{V}} \\ 0 & \text { otherwise }\end{cases}
$$


The (possibly) constrained departure time is the unconstrained departure time plus any extra non-control holding time (i.e. blocked time) required to prevent overtaking.

$$
\bar{d}_{v, s}=d_{v, s}+\bar{h}_{v, s} \quad \forall v \in V \quad \forall s \in S
$$

A vehicle's dwell time can be modeled with alightings and boardings happening in series or in parallel. Boarding and alighting times are modeled as linear functions of the number of boarding $\bar{\beta}_{v, s}$ and alighting $\alpha_{v, s}$ passengers, respectively. Constant boarding and alighting times per passenger, $\tau_{b}$ and $\tau_{a}$, are assumed. Bus systems in which boarding and alighting passengers use different doors are modeled with a parallel process, in which case dwell time is the greater of the boarding and alighting times.

$$
\delta_{v, s}=\max \left(\tau_{b} \bar{\beta}_{v, s}, \tau_{a} \alpha_{v, s}\right) \quad \forall v \in V \quad \forall s \in S
$$

In typical rail systems, the boarding process begins after the alighting process finishes, in which case dwell time is the sum of the boarding and alighting times.

$$
\delta_{v, s}=\tau_{b} \bar{\beta}_{v, s}+\tau_{a} \alpha_{v, s} \quad \forall v \in V \quad \forall s \in S
$$

Demand is modeled at the origin-destination pair level $\left(s_{b}\right.$ to $\left.s_{a}\right)$. For the first visit to each stop, it includes passengers who are already at the stop at the beginning of the optimization horizon, $\iota_{s_{b}, s_{a}}^{\prime}$. For subsequent visits, it includes passengers who were unable to board the preceding vehicle because it was full, $\pi_{v-1, s_{b}, s_{a}}$. The number of arriving passengers is determined by integrating timedependent passenger arrival rate functions $\lambda_{s_{b}, s_{a}}(t)$. The number of passengers waiting to board per origin-destination pair is given by

$$
\beta_{v, s_{b}, s_{a}}=\left\{\begin{array}{lll}
\iota_{s_{b}, s_{a}}^{\prime}+\int_{t_{0}}^{d_{v, s_{b}}} \lambda_{s_{b}, s_{a}}(t) \mathrm{d} t & v=g_{s_{b}} & \forall s_{b} \in S \quad \forall s_{a} \in S \\
\pi_{v-1, s_{b}, s_{a}}+\int_{d_{v-1, s_{b}}}^{d_{v, s_{b}}} \lambda_{s_{b}, s_{a}}(t) \mathrm{d} t & \forall v \in V & \forall s_{b} \in\left[e_{v-1}+1, e_{v}\right] \\
& \forall s_{a} \in S &
\end{array}\right.
$$

where vehicle $g_{s_{b}}$ is the first to visit stop $s_{b}$ in the horizon. The total number of waiting passengers is

$$
\beta_{v, s}=\sum_{s_{a} \in S} \beta_{v, s, s_{a}} \quad \forall v \in V \quad \forall s \in S
$$

The actual number of boardings cannot exceed the remaining capacity, which is determined by subtracting load $l_{v, s}$ and adding alightings to capacity $k$.

$$
\bar{\beta}_{v, s}=\min \left(\beta_{v, s}, k-l_{v, s}+\alpha_{v, s}\right) \quad \forall v \in V \quad \forall s \in S
$$

When the number of passengers who want to board exceeds the remaining capacity, it is assumed that all passengers, regardless of destination, are equally 
likely to board.

$$
\bar{\beta}_{v, s_{b}, s_{a}}=\frac{\bar{\beta}_{v, s_{b}}}{\beta_{v, s_{b}}} \beta_{v, s_{b}, s_{a}} \quad \forall v \in V \quad \forall s_{b} \in S \quad \forall s_{a} \in S
$$

The number of passengers $\pi_{v, s_{b}, s_{a}}$ prevented from boarding (by origin-destination pair) is the difference between passengers waiting and those who board.

$$
\pi_{v, s_{b}, s_{a}}=\beta_{v, s_{b}, s_{a}}-\bar{\beta}_{v, s_{b}, s_{a}} \quad \forall v \in V \quad \forall s_{b} \in S \quad \forall s_{a} \in S
$$

The number of passengers prevented from boarding vehicle $v$ at stop $s$ is

$$
\pi_{v, s}=\beta_{v, s}-\bar{\beta}_{v, s}=\sum_{s_{a} \in S} \pi_{v, s, s_{a}} \quad \forall v \in V \quad \forall s \in S
$$

Alightings come not only from passengers who board during the optimization horizon but also from passengers who are already in the vehicle at the beginning of the optimization horizon. We assume that all passengers in a vehicle at the last stop alight. Thus the number of alighting passengers includes passengers initially in the vehicle starting at the first stop the vehicle visits and up to the last stop. We assume that there is no demand for travel from a stop to the same stop, i.e. $\beta_{v, s, s}=0$ for every vehicle and stop.

$$
\alpha_{v, s_{a}}=\left\{\begin{array}{lll}
l_{v, s_{a}}^{\prime}+\sum_{s_{b}=e_{v}+1}^{s_{a}} \bar{\beta}_{v, s_{b}, s_{a}} & \forall v \in V & \forall s_{a} \in\left[e_{v}+1, n_{S}\right] \\
\sum_{s_{b}=e_{v}+1}^{s_{a}} \bar{\beta}_{v, s_{b}, s_{a}} & \forall v \in V & \forall s_{a} \in\left[1, e_{v}\right]
\end{array}\right.
$$

Vehicle loads $l_{v, s}$ upon arrival at the first stop are known as part of the initial state. Vehicle loads upon arrival at subsequent stops are obtained recursively.

$$
l_{v, s}=\left\{\begin{array}{lll}
\sum_{s_{a} \in S} l_{v, s_{a}}^{\prime} & \forall v \in V & s=e_{v}+1 \\
l_{v, s-1}+\bar{\beta}_{v, s-1}-\alpha_{v, s-1} & \forall v \in V & \forall s \in\left[e_{v}+2, e_{v}\right]
\end{array}\right.
$$

The set of equations in this section can be evaluated recursively to obtain a deterministic forecast of how the system will evolve over the next cycle. Specifically, the arrival time, arrival load, and departure time of each vehicle are estimated for each vehicle-stop combination. The prediction will generally differ from the actual future states due to stochasticity and model simplifications.

Each evaluation of the performance model requires multiple evaluations of running time functions $r_{s}(t)$ and integrations of passenger arrival rate functions $\lambda_{s_{b}, s_{a}}(t)$. The computational complexity of these tasks depends on the functional forms and algorithms employed. In this research, piecewise-linear functions are used for dynamic running times and demand. Bisection search and linear interpolation are used for evaluation, while bisection search and trapezoidal integration are used for integration. Integrals are pre-calculated for each 
point, leaving only the between-points portion of the function to integrate onthe-fly.

The number of passengers inside vehicles and at stops (by origin-destination pair) at the beginning of the prediction horizon (denoted by $l_{v, s_{a}}^{\prime}$ and $\iota_{s_{b}, s_{a}}^{\prime}$, respectively) are estimated using equations (16) through (23), from actual vehicle passage times (before $t_{0}$ ) and dynamic demand functions. Whenever a vehicle visits a stop, the estimated number of passengers originating at the current stop and destined for downstream stops is determined using the dynamic passenger arrival rate and the time since the previous vehicle departure, while the estimated number of passengers destined for the current stop is set to zero (because they have alighted). The initial number of passengers at stops includes the estimated number of passengers previously left behind by full vehicles.

\subsection{Cost Model}

The cost model determines mean cost per passenger using the prediction information from the performance model. Cost is based on waiting time and in-vehicle delay due to holding.

In-vehicle delay due to holding is the product of the number of throughpassengers in the vehicle and the holding time. The total in-vehicle delay over the horizon is given by

$$
W_{V}=\sum_{v \in V} \sum_{s \in S}\left(l_{v, s}-\alpha_{v, s}\right) h_{v, s}
$$

Waiting time has four components corresponding to whom is affected: passengers who arrive before the start of the prediction horizon, passengers who arrive after the start of the prediction horizon and are served by the first vehicle to arrive at the stop in the prediction horizon, passengers who arrive between successive vehicle visits within the prediction horizon, and passengers who cannot board the first vehicle to visit their origin stop after their arrival because it was full.

$$
W_{S}=W_{S_{0}}+W_{S_{1}}+W_{S_{2}}+W_{S_{3}}
$$

For passengers who arrived before the start of the prediction horizon, we consider only their waiting time within the prediction horizon, given by

$$
W_{S_{0}}=\sum_{s_{b} \in S}\left[\left(\sum_{s_{a} \in S} \iota_{s_{b}, s_{a}}^{\prime}\right)\left(\bar{d}_{g_{s_{b}}, s_{b}}-t_{0}\right)\right]
$$

where $\bar{d}_{g_{s_{b}}, s_{b}}$ is the time of the first departure from stop $s_{b}$ in the prediction

horizon. For passengers arriving between $t_{0}$ and the first departure, waiting time depends on the arrival rate of passengers.

$$
W_{S_{1}}=\sum_{s_{b} \in S} \sum_{s_{a} \in S} \int_{t_{0}}^{\bar{d}_{g_{s_{b}}, s_{b}}} \lambda_{s_{b}, s_{a}}(t)\left(\bar{d}_{g_{s_{b}}, s_{b}}-t\right) \mathrm{d} t
$$


In order to reduce computational complexity, we approximate mean passenger waiting times by assuming uniform passenger arrivals over the headway.

$$
W_{S_{1}} \approx \sum_{s_{b} \in S} \sum_{s_{a} \in S}\left(\int_{t_{0}}^{\bar{d}_{g_{s_{b}}, s_{b}}} \lambda_{s_{b}, s_{a}}(t) \mathrm{d} t\right) \frac{\bar{d}_{g_{s_{b}}, s_{b}}-t_{0}}{2}
$$

This allows reusing the integral computed in (16) of the performance model. The approximation is reasonable for high-frequency systems in which mean passenger arrival rates change gradually.

The waiting time of passengers who arrive in headways contained in the prediction horizon depends on both passenger arrival rates and the departure times that determine the headway. Again, mean passenger waiting times are approximated by assuming uniform passenger arrivals over the headway.

$$
\begin{aligned}
W_{S_{2}} & =\sum_{v \in V} \sum_{s_{b}=e_{v-1}+1}^{e_{v}} \sum_{s_{a} \in S} \int_{\bar{d}_{v-1, s_{b}}}^{\bar{d}_{v, s_{b}}} \lambda_{s_{b}, s_{a}}(t)\left(\bar{d}_{v, s_{b}}-t\right) \mathrm{d} t \\
W_{S_{2}} & \approx \sum_{v \in V} \sum_{s_{b}=e_{v-1}+1}^{e_{v}} \sum_{s_{a} \in S}\left(\int_{\bar{d}_{v-1, s_{b}}}^{\bar{d}_{v, s_{b}}} \lambda_{s_{b}, s_{a}}(t) \mathrm{d} t\right) \frac{\bar{d}_{v, s_{b}}-\bar{d}_{v-1, s_{b}}}{2}
\end{aligned}
$$

Some passengers find that the first vehicle to arrive at their stop is full. They are unable to board that vehicle and must wait for the next vehicle with available space. Their additional waiting time is given by

$$
W_{S_{3}}=\sum_{v \in V} \sum_{s_{b}=e_{v-1}+1}^{e_{v}} \pi_{v-1, s_{b}}\left(\bar{d}_{v, s_{b}}-\bar{d}_{v-1, s_{b}}\right)
$$

The total number of passengers who board over the entire prediction horizon is given by

$$
P=\sum_{v \in V} \sum_{s \in S} \bar{\beta}_{v, s}
$$

\subsection{Control Strategy}

Every time a vehicle arrives at a control point (i.e. a terminal or stop at which holding is allowed), the model presented in this section is used to optimize holding times for all vehicles at all stops over the rolling prediction horizon. (An alternative approach not used in this research is to optimize holding times periodically, e.g. once per minute, and use the most recently optimized holding

times for all vehicles.) The holding time suggested by the optimization algorithm for the vehicle that triggered the modeling-optimization event at its current stop is implemented.

\subsection{Dimensionality Reduction}

The complexity of the optimization problem is a function of the product of the number of control points and the number of controlled vehicles. Since the 
optimization model is called frequently, it is important to control the problem size. Optimization times may become prohibitive for instances with many control points and a large fleet. We have had success implementing optimizationbased control in such cases by reducing the number of stops that are control points (e.g. control points every 5 stops) and also optimizing the holding times of a control set $V_{C} \subset V$ of 5 vehicles, consisting of the vehicle that triggered the optimization event, the two preceding vehicles, and the two following vehicles.

When a reduced vehicle control set is used, optimization policy must be approximated for vehicles not in the control set; otherwise optimization will produce plans assuming that other vehicles are not controlled, which could be inconsistent with what would happen at a future optimization event that does hold those vehicles. Past research (e.g. see Eberlein et al., 2001), as well as well as our own experiments, show that optimization-based strategies usually lead to even headways. We have had good results using the even headway strategy (presented in Section 4) to approximate the optimization policy for vehicles not in the control set. It is also possible to use previously optimized holding times if they are available.

In the case study presented in Section 4 , there are 8 control points and 10 vehicles, which makes 80 decision variables. Allowing holding at a reduced set of stops ( 8 out of 40 ) reduces the problem size sufficiently that there is no need to also reduce the set of controlled vehicles.

\section{Application}

The proposed framework is evaluated in a simulation of high-frequency bus service. This allows the comparison of different strategies in a controlled manner, where differences in performance between cases having a particular set of demand and running time dynamics are mostly due to differences in control strategies. The simulation model is event-based. Within each replication, events such as passenger and vehicle arrivals at stops and boarding and alighting activity are processed chronologically. (Sánchez-Martínez, 2012)

The simulated transit system is a simple route with 20 stops in each direction. Vehicles have capacity for 60 passengers. Boarding and alighting times per passenger are deterministic with $\tau_{b}=\tau_{a}=2$ seconds, and the boarding and alighting processes happen in parallel. Service operates with 10 vehicles. Stochastic running times between stops are drawn from a log-normal distribution with a mean of 60 seconds and a coefficient of variation of 0.4 , though the distribution is shifted by dynamics as described later. The use of log-normal distributions to model running times is common practice (Zhao et al., 2003, Cats et al., 2011, Dessouky et al., 2003, and Delgado et al., 2012). Cats et al. (2011) analyzed empirical segment running time data and found that all links studied follow a log-normal distribution based on Chi-squared and Kolmogorov-Smirnov goodness-of-fit tests at a confidence level of $95 \%$.

The passenger arrival process is Poisson, with the same mean arrival rate for all origin-destination pairs in each direction. This demand specification results in (for each direction) the first stop having the highest number of passengers 
boarding, the last stop having the highest number of passengers alighting, and the middle stop having the highest vehicle loads. Two demand levels are considered: low crowding and high crowding. The base mean arrival rate in the first direction is set such that (if vehicles arrive every 5 minutes) peak loads reach $75 \%$ of capacity in high crowding cases and $25 \%$ of capacity in low crowding cases. The passenger arrival rates in the second direction are half those in the first direction.

The simulation starts with all vehicles at a terminal. Vehicles are dispatched at even headways, and passengers begin to arrive at stops shortly before each stop's first vehicle visit. Running times are deterministic for the first hour in order to discourage bunching in the absence of control. When all vehicles have been dispatched, almost an hour after the beginning of the simulation, running times become stochastic and control begins. The analysis period begins two hours into the simulation and lasts two hours. All passengers who arrive at their origin stop during the analysis period are included when calculating performance measures, even if their trips end after the analysis period.

We test the effectiveness of the dynamic control strategy in six different cases involving different dynamics and crowding levels, as shown in Table 1. There are low and high crowding variants of dynamic running times and demand, dynamic running times but static demand, and static running times but dynamic demand.

Dynamics in running times and demand are introduced by a dynamic factor $\phi(s, t)$ used to transform base mean running times and mean passenger arrival rates in the first direction. The factor is defined as follows:

$$
\phi(s, t)= \begin{cases}1.0+2.0\left(t-r^{*}(s-1)-2.0\right) & \text { for } 2.0 \leq t-r^{*}(s-1)<2.5 \\ 2.0-2.0\left(t-r^{*}(s-1)-2.5\right) & \text { for } 2.5 \leq t-r^{*}(s-1)<3.0 \\ 1.0 & \text { otherwise }\end{cases}
$$

where $t$, time, and $r^{*}$, base running time between stops, are expressed in hours. Figure 3 shows the dynamic factor applied to the rate of passengers arriving at the first stop and running times between the first and second stops. In this case, where $s=1$, running times and demand begin increasing at the beginning of the analysis period, peak at $t=2.5$ hours, and return to their base values at $t=3.0$ hours, one hour into the analysis period. The $r^{*}(s-1)$ term introduces a lag in the transformation for stops after the first. For example, the rate of passengers arriving at the tenth stop peaks 9 minutes after $t=2.5$ hours. The lag introduces correlation of running times and demand across stops in the first direction, making some vehicles experience near peak running times and demand throughout the entire run. The dynamic transformation shifts running time distributions without affecting their variance. In cases having dynamic demand, the dynamic transformation affects the mean arrival rates $\lambda_{s_{b}, s_{a}}(t)$ governing the Poisson process. In the two cases having dynamic demand with high crowding, vehicles reach capacity and some passengers are unable to board the first vehicle.

Using this setup, we independently simulate service with the static and dy- 


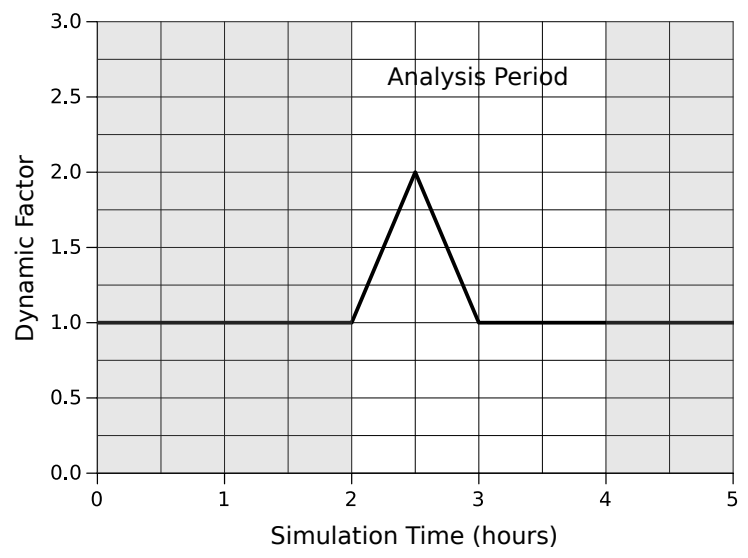

Figure 3: Dynamic Factor for the First Stop

namic control using the optimization model formulated in Section 3. In addition to the static and dynamic optimization-based strategies, we also evaluate service with two heuristic control strategies in order to have a point of reference for the performance of the optimization-based strategies.

The following is a list of the strategies considered:

TH (Target Headway). Hold vehicle $v$ at control point $s$ to ensure preceding headways are never less than a prescribed target headway $H$.

$$
h_{v, s}=\max \left(0, H-\left(d_{v, s}-d_{v-1, s}\right)\right)
$$

where $d_{v, s}$ is the departure time of the vehicle being controlled if no holding is applied, and $d_{v-1, s}$ is the time of the previous departure from the control point. The performance of this strategy depends on the selection of $H$. Optimum target headways for each case are found by simulating service with a range of target headways and selecting the ones yielding lowest mean passenger cost for the analysis period. Table 1 shows optimum target headways for each case. This control strategy gives an upper bound on the performance (lower bound on the cost) that can be attained with a constant target headway strategy, though it might be possible to improve performance further with time-varying target headways. Since the other strategies are evaluated by comparison to this strategy, the optimization of target headways reduces the reported effectiveness of the other strategies. This approach differs from the one more commonly followed in the literature (e.g. Delgado et al., 2012), in which a non-optimized schedule headway (or in some cases no control at all) is used as a base case that favors proposed control strategies.

EH (Even Headway). Hold with the aim of equalizing the preceding and following headways. When a vehicle is ready to depart, its preceding and following 
Table 1: Cases

\begin{tabular}{cccc}
\hline Running Times & Demand & Crowding & Optimum Target Headway (min) \\
\hline dynamic & dynamic & high & 4.5 \\
dynamic & dynamic & low & 4.6 \\
dynamic & static & high & 4.7 \\
dynamic & static & low & 4.6 \\
static & dynamic & high & 4.5 \\
static & dynamic & low & 4.1 \\
\hline
\end{tabular}

headways are estimated. If the following headway is longer, the vehicle is held by half the difference between the two headways, with the intent of making the vehicle depart when the preceding and following headways are equal. Strategies like this one have been implemented before using information about past departure times to obtain headway estimates. Our implementation uses the performance model to predict headways with dynamic information. Specifically, the model forecasts departure times of the following vehicle from the control vehicle's current stop $\bar{d}_{v+1, s}$ (to obtain following headway), and of the control vehicle from the preceding vehicle's most recently departed stop $\bar{d}_{v, e_{v-1}}$ (to obtain preceding headway), as given by

$$
h_{v, s}=\max \left(0, \frac{\bar{d}_{v+1, s}-d_{v+1, e_{v+1}}^{\prime}-\bar{d}_{v, e_{v-1}}+t_{0}}{2}\right)
$$

OS (Optimization with Static inputs). Hold at selected control points according to the suggestion from the rolling horizon optimization model, with running times and demand inputs defined as period-specific time-independent constants, similar to Delgado et al. (2012). Three periods are defined: one covering the time during which dynamics are in effect and two covering times before and after, when no dynamics are in effect.

OD (Optimization with Dynamic inputs). Hold at selected control points according to the suggestion from the rolling horizon optimization model, with running times and demand inputs defined as time-dependent functions.

\section{Results and Discussion}

The detailed output of the simulation model includes vehicle trajectories and arrival time at the origin, boarding time, and arrival time at the destination for each passenger. From this we can derive performance measures such as waiting time, in-vehicle time, trip time, vehicle loads, holding times, headways, and number of passengers at stops. A probability density function is obtained for each of these performance measures, from which statistics such as mean, standard deviation, and percentiles can be calculated. The principal performance measure of the following analysis is passenger cost.

The trips of passengers who arrive at their origin stop in the analysis period are used to calculate performance measures. Passenger cost and excess waiting 


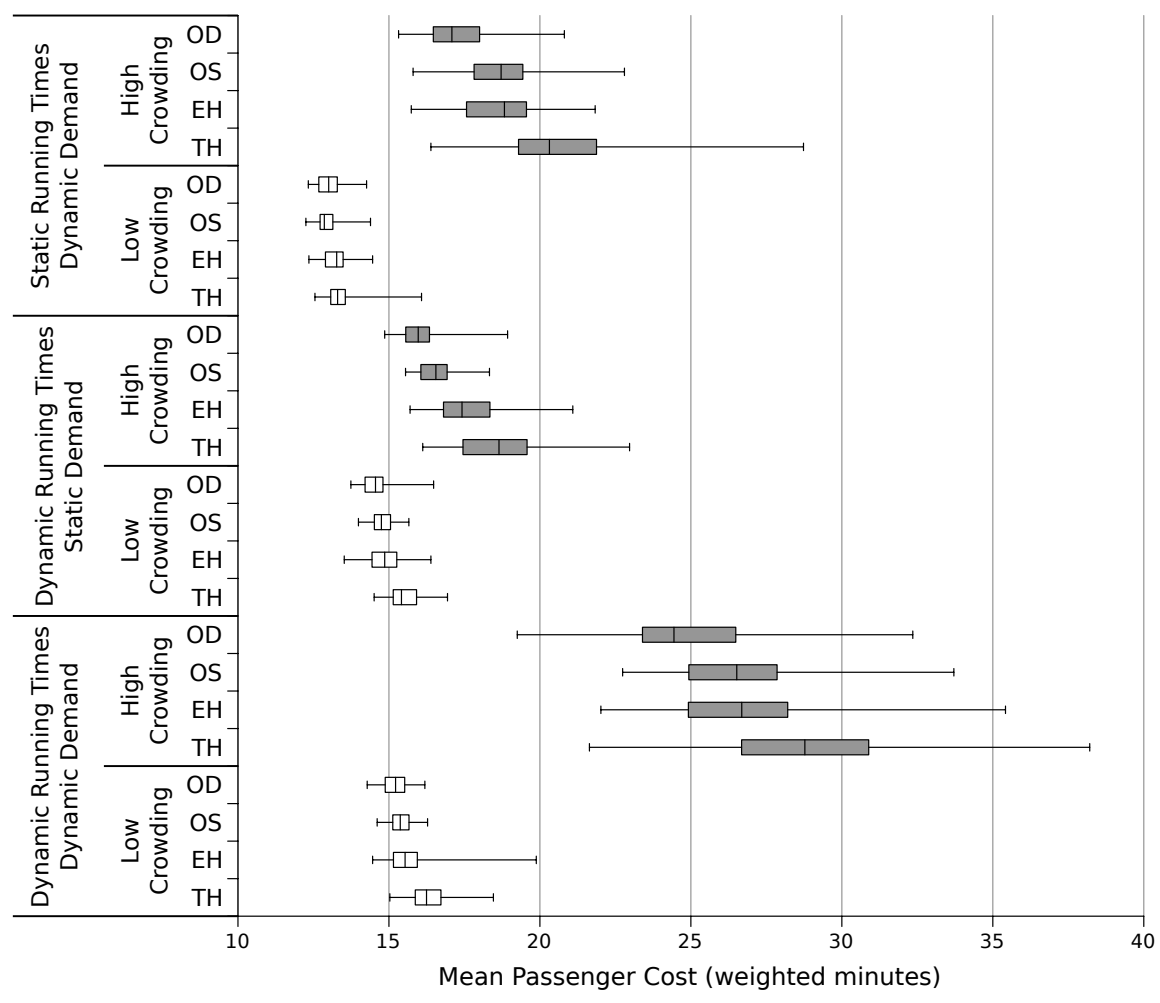

Figure 4: Distributions of mean passenger cost

time (waiting time in excess of half the scheduled headway, which is 5 minutes in all cases) are calculated for each passenger, and the mean cost and excess waiting time across passengers are obtained for each replication. 60 replications were run for each experiment. These results are discussed in Section 5.1. Analysis of headways is presented in Section 5.2, and analysis of stop crowding in Section 5.3. Section 5.4 presents an analysis of the sensitivity of performance improvements to stochasticity. Section 5.5 explores the value of modeling dynamics when the current state is known perfectly. Section 5.7 presents a comparison of computation times for the static and dynamic optimization-based control strategies.

\subsection{Passenger Cost}

Figure 4 shows a box-and-whisker plot of mean passenger cost. On the basis of mean passenger cost across replications, $\mathrm{EH}$ is consistently better than $\mathrm{TH}$, OS is either better than or similar to EH, and OD is consistently better than or similar to OS.

In the case of dynamic running times and demand and low crowding, small 
decreases in mean passenger cost are observed going from EH to OS and from OS to OD, though the $90^{\text {th }}$ percentile cost decreases by 0.43 weighted minutes going from EH to OS. In case of dynamic running times and demand and high crowding, passenger cost distributions are similar for the EH and OS strategies, but a significant cost decrease (1.71 weighted minutes or $6.4 \%$ ) is observed going from OS to OD.

In the case of dynamic running times, static demand, and low crowding, mean passenger costs are similar for the EH and OS strategies, and a very small cost decrease ( 0.21 weighted minutes or $1.4 \%$ ) is seen going from OS to OD. Although the maximum cost is greater with OD than OS, the $90^{\text {th }}$ percentile cost is 0.34 weighted minutes less with OD than OS. In the case of dynamic running times, static demand, and high crowding, mean passenger cost decreases by 1.12 weighted minutes $(6.3 \%)$ going from $\mathrm{EH}$ to OS, and by 0.51 weighted minutes $(3.1 \%)$ going from OS to OD. The $90^{\text {th }}$ percentile cost decreases by 0.46 minutes going from OS to OD. These results suggest that moderate performance improvements are achieved by the OD strategy when running times are dynamic, even if passengers are not being left behind due to overcrowding.

In the case of static running times, dynamic demand, and low crowding, a small decrease in mean passenger cost is observed going from EH to OS, and there is no significant difference between the OS and OD cost distributions. This suggests that when dynamics are present only in demand and in the absence of overcrowding, there is no significant benefit to optimizing holding times with dynamic running times and demand. This result is consistent with the finding of Eberlein et al. (2001) that holding policies are not highly sensitive to demand patterns; our results suggest that they are also not sensitive to changes in demand over time, unless vehicle capacity is exceeded.

In the case of static running times, dynamic demand, and high crowding, the EH and OS cost distributions are similar, but mean cost decreases by 1.34 weighted minutes $(7.2 \%)$ and the $90^{\text {th }}$ percentile cost decreases by 1.51 minutes going from OS to OD.

Large performance improvements are seen only in the cases involving dynamic demand and high crowding, which are the two cases having the highest number of passengers left behind. These cases have $6.4 \%$ and $7.2 \%$ decreases in mean passenger cost going from OS to OD. This suggests that the greatest benefits of optimizing with dynamic running times and demand can be expected when, due to dynamics in running time or demand, a significant fraction of passengers have to wait for more than one vehicle. This is due to the dynamic model's ability to predict when vehicles will be loaded to capacity, a critical factor in determining optimum holding policies, since it is not effective to hold vehicles that will fill up. The OS strategy is equally able to predict when capacities are reached if running times or demand do not vary significantly over the rolling optimization horizon.

Observed differences in excess waiting time closely parallel those in mean passenger cost. Excess waiting time calculations assume a scheduled headway of 5 minutes in all cases. Decreases in excess waiting time of $15.8 \%$ and $25.0 \%$ going from OS to OD are observed for the cases involving dynamic demand and 
Table 2: Headways at First Stop

\begin{tabular}{llllrr}
\hline Running Times & Demand & Crowding & Strategy & Mean & Standard Deviation \\
\hline \multirow{2}{*}{ Dynamic } & Dynamic & Low & TH & 5.16 & 2.52 \\
& & & EH & 4.79 & 2.46 \\
& & & OS & 5.04 & 1.96 \\
& & & OD & 4.93 & 2.07 \\
\hline Dynamic & Dynamic & \multirow{2}{*}{ High } & TH & 5.73 & 2.78 \\
& & & EH & 5.46 & 2.67 \\
& & & OS & 5.47 & 2.74 \\
& & & OD & 5.43 & 2.97 \\
\hline Dynamic & Static & \multirow{2}{*}{ Low } & TH & 5.11 & 2.30 \\
& & & EH & 4.76 & 2.31 \\
& & & OS & 4.95 & 1.98 \\
& & & OD & 4.90 & 1.94 \\
\hline \multirow{2}{*}{ Dynamic } & Static & \multirow{2}{*}{ High } & TH & 5.78 & 2.45 \\
& & & EH & 5.44 & 2.58 \\
& & & OS & 5.69 & 1.68 \\
& & & OD & 5.54 & 1.51 \\
\hline \multirow{2}{*}{ Static } & Dynamic & Low & TH & 4.52 & 1.71 \\
& & & EH & 4.28 & 2.04 \\
& & & OS & 4.42 & 1.59 \\
& & & OD & 4.40 & 1.71 \\
\hline \multirow{2}{*}{ Static } & Dynamic & High & TH & 5.13 & 1.43 \\
& & & EH & 4.83 & 1.91 \\
& & & OS & 5.02 & 1.55 \\
& & & OD & 4.97 & 2.11 \\
\hline & & & & &
\end{tabular}

high crowding.

\subsection{Headways}

Table 2 shows the mean and standard deviation of headways at the first inbound stop. In all cases, the TH strategy yields the greatest mean headways. Noting that this strategy also yields the greatest mean passenger cost, we observe that performance improvements are possible with more sophisticated holding strategies. The time vehicles spend holding is not productive, and greater overall holding times lead to less frequent service and greater passenger waiting times. However, not holding enough may lead to bunching that is difficult to recover from, which is another way in which productivity can suffer. While the performance advantage of $\mathrm{EH}$ over $\mathrm{TH}$ stems at least in part from running more frequent service, mean headways increase or remain similar when going from $\mathrm{EH}$ to OS in all cases. This suggests that the advantage of OS over EH is not due to running more frequent service. Moreover, mean headways decrease by only 0.04 and 0.05 minutes going from OS to OD in the two cases having dynamic demand and high crowding, suggesting that the two largest performance improvements going from OS to OD are not significantly due to running more frequent service.

One might expect that holding optimization necessarily involves a trade-off between frequency of service and headway regularity, since more holding allows 


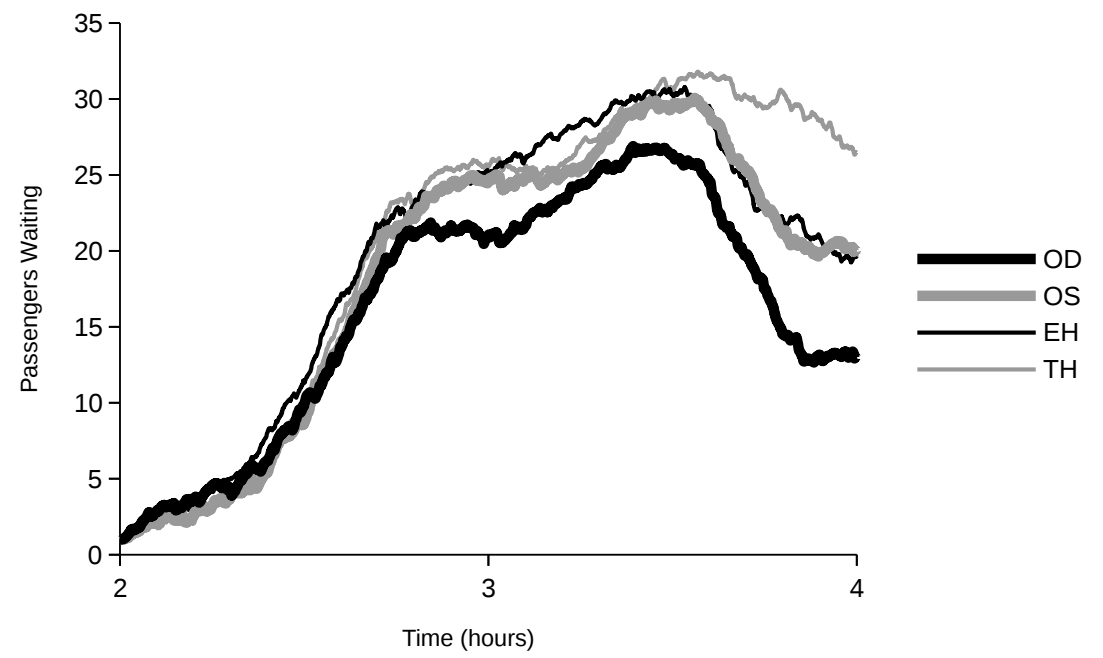

Figure 5: Stop Crowding at the Maximum Load Point

decreasing headway variability. The results in Table 2 show this is not always the case. For instance, in the case of dynamic running times and demand and low crowding, the OD strategy reduces both the mean and the standard deviation of headways with respect to the TH strategy. This indicates that some strategies choose holding times more effectively.

One might also expect that for a given mean headway, lower headway variabilities lead to better performance. This notion, captured in the commonly used equation expressing expected waiting time in terms of headway mean and standard deviation, assumes that passengers are not left behind by (full) vehicles and that headway variability is entirely random (rather than systematically time-dependent). In the two cases with the greatest decreases in mean passenger cost going from OS to OD, which are also the two cases with dynamic demand and high crowding, mean headways are similar but headway variability increases going from OS to OD. In these cases a significant number of passengers are left behind, so the best holding policies are not necessarily those that balance headways. Indeed, there may be situations in which strategic bunching is desirable for a period of time to manage overcrowding.

\subsection{Crowding at Stops}

The number of passengers waiting at the maximum load point (the midpoint stop in the first direction) was analyzed in the case of dynamic running times and demand and high crowding, and is shown in Figure 5 for the different control strategies. 


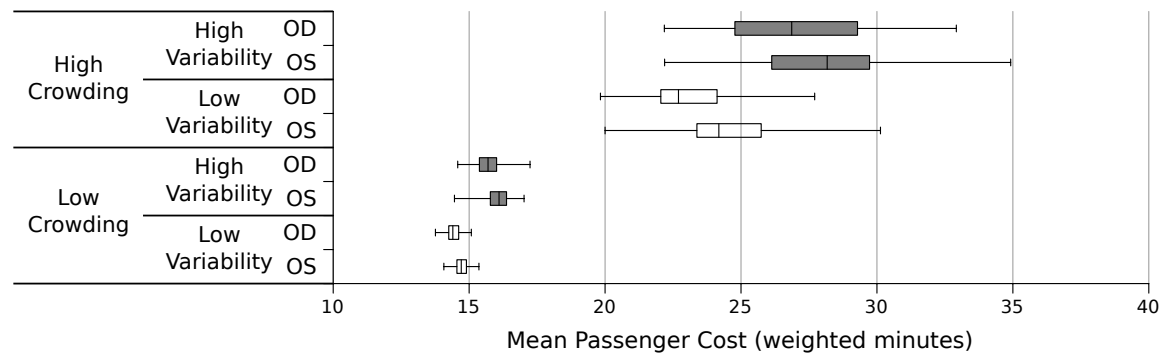

Figure 6: Distributions of mean passenger cost for low and high running time variability

The OD strategy leads to the lowest stop crowding throughout most of the analysis period, ending at about 13 passengers compared to about 20 passengers for the EH and OS strategies. The difference in number of passengers at this time is as large between OS and OD as between TH and EH. This suggests that the OD strategy is more effective in controlling overcrowding at stops in systems running at capacity with significant running time and demand dynamics.

\subsection{Sensitivity to Stochasticity}

The earlier discussion shows that the OD strategy can lead to improved performance in some cases. There are at least two possible reasons for this. First, since the OD strategy can predict future system states more accurately, it may be able to generate preemptive control policies that prepare the system for delays or surges in demand before these reach a critical level. Second, although the forecasting model underlying the OS and OD strategies is deterministic, the OD strategy may be able to react to unpredictable stochasticity more effectively than the OS strategy by considering dynamics of running times and demand. In order to understand which of these plays the greater role, we conduct a sensitivity analysis of system performance using the OS and OD holding strategies under low and high running time variability. The analysis focuses on the two cases having dynamic running times and demand (with low and high crowding).

In all previous cases, running times between consecutive stops are drawn from a log-normal distribution with a mean of 60 seconds and a coefficient of variation of 0.4 (shifted for dynamics). We introduce low and high variability variants that instead have coefficients of variation of 0.2 and 0.6 , respectively. In all cases, demand follows the same Poisson process as before. Figure 6 shows mean passenger cost distributions in a box and whisker plot.

Both mean passenger costs and variability in these means across replications increase with running time variability. As before, the OD strategy yields lower mean passenger costs than the OS strategy, and the improvement is small in cases with low crowding and significant in cases with high crowding. Going from OS to OD, mean passenger costs decrease by 0.28 weighted minutes in the low crowding, low variability case, 0.33 weighted minutes in the low crowding, 
high variability case, 1.31 weighted minutes in the high crowding, low variability case, and 1.20 minutes in the high crowding, high variability case. For low crowding, the improvement is 0.05 weighted minutes greater in the high variability case than the low variability case. For high crowding, the improvement is 0.11 weighted minutes less in the high variability case than the low variability case. The small magnitude of these two differences suggests that performance improvements going from OS to OD are robust to changes in system stochasticity, and that most of the improvements come not from the OD strategy reacting with greater aptitude to unpredictable stochasticity, but from its ability to generate preemptive policies in light of forecast dynamics in running times and demand.

\subsection{Sensitivity to Current State Information Accuracy}

Since the OD strategy incorporates time-varying running times and demand, it is better able to estimate the current system state as well as predict future states under a variety of control policy scenarios. The model used for holding time optimization takes inputs that specify both the current system state and the running time and demand functions that govern how the system is expected to evolve. For instance, the number of passengers initially waiting at stops $\left(\iota_{s_{b}, s_{a}}^{\prime}\right)$ or inside vehicles $\left(l_{v, s_{a}}^{\prime}\right)$, by origin-destination pair, are given to the optimization model. The OD strategy can estimate these quantities better because it has more accurate descriptions of passenger arrival rates over time.

We have seen that the OD strategy improves performance in some cases, but the question remains: to what extent do performance benefits stem from the ability to estimate current system state more accurately rather than from the ability to predict future system states with dynamic running times and demand? If the benefit came mostly from the former, then performance under the OS strategy could be improved with the use of passenger counting technologies. To investigate, we evaluate operations of the case of dynamic running times and demand and high crowding using the OS and OD strategies, but replacing the estimation of number of passengers at stops and in vehicles by the true values known to the simulation model. In reality it would be difficult to obtain this information, but the result of this experiment allows us to understand the relative importance of accuracy of current vs. future system states.

Figure 7 shows mean passenger cost distributions for the case of dynamic running times and demand and high crowding in three different scenarios having different information about the current state. In the first case, the number of passengers is estimated both in vehicles and at stops. This is the base case presented in Section 5.1, repeated here for ease of comparison. In the second case, the number of passengers in vehicles (by destination) is known perfectly but the number of passengers at stops (by destination) is estimated. In the third case, the number of passengers is known perfectly both in vehicles and at stops.

The improvement going from the OS strategy to the OD strategy is 1.71 weighted minutes in the base case, 1.31 weighted minutes in the case of known vehicle loads but estimated number of passengers at stops, and 0.74 weighted 


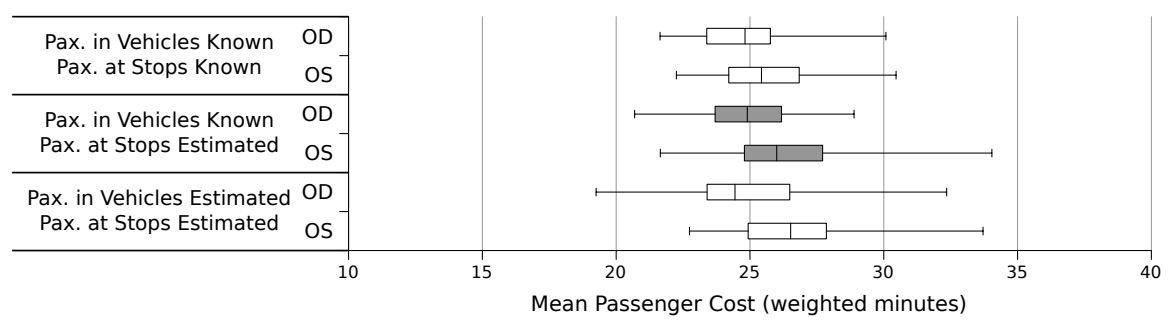

Figure 7: Distributions of mean passenger cost with perfect current state information

minutes in the case of known vehicle loads and number of passengers at stops. As expected, the improvement decreases as more current state information is known instead of estimated, because the information accuracy gap closes. The 0.40 weighted minutes improvement gained by knowing the number of passengers initially in vehicles is $24 \%$ of the 1.71 weighted minutes improvement in the base case. The 0.97 weighted minutes improvement gained by knowing the number of passengers initially in vehicles and at stops is $57 \%$ of the 1.71 weighted minutes improvement in the base case. In other words, more than half of the OD strategy's advantage appears to come from estimating the current state, rather than future states, more accurately.

Focusing on the scenarios with the OD control strategy, there is negligible performance difference between the base case and the case of known vehicle loads but estimated number of passengers at stops, and a very small improvement of 0.13 weighted minutes going from that to the case of known number of passengers in vehicles and at stops. This indicates that the OD strategy's current state demand estimates are sufficiently accurate for this application.

\subsection{Sensitivity to a Holding Time Adjustment Factor}

Thus far the discussion has focused on results of simulation runs in which vehicles hold as long as suggested by the optimization model. In this section we consider what happens when vehicle holds are shorter or longer. This question is relevant when vehicles are driven by humans who for various reasons may not be able or willing to hold for the exact times suggested by the optimization model. Moreover, Delgado et al. (2012) find that holding between 0.25 and 0.75 of the time suggested by their optimization model improves results significantly; they attribute this to the possibility that the model overreacts because it does not capture stochasticity. To investigate how an adjustment factor affects results, we evaluate operations of the case of dynamic running times and demand and high crowding using the OS and OD strategies, applying adjustment factors ranging from 0.25 to 1.75 , in increments of 0.25 . The optimization model runs as before, assuming vehicles will hold for the exact times suggested, but the simulation model holds for the adjusted time.

Figure 8 shows how mean passenger cost responds to adjusted holding times. Performance is relatively robust with both strategies for adjustment factors be- 


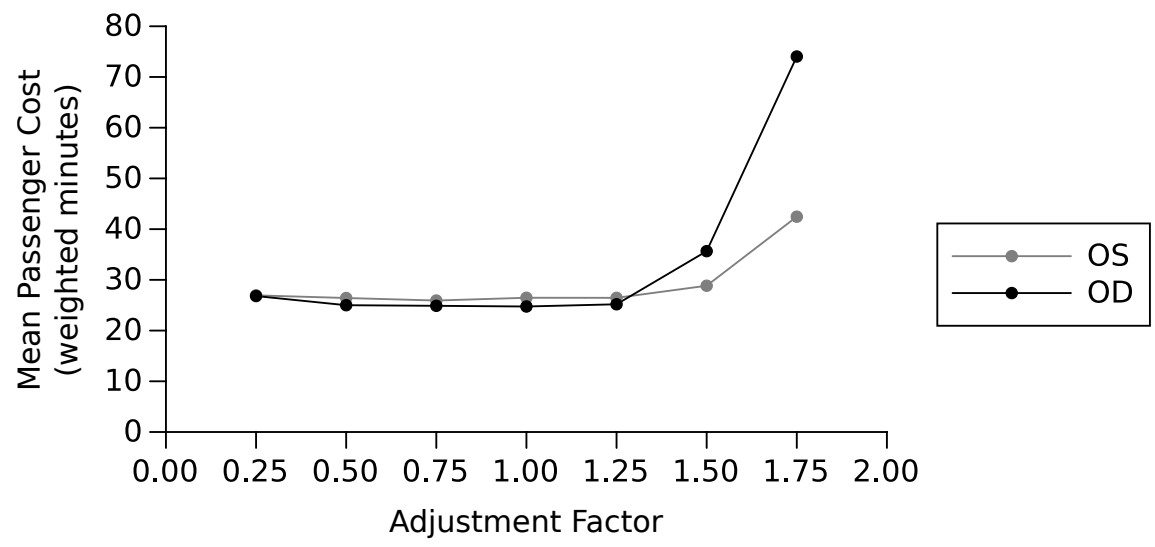

Figure 8: Effect of Adjusting Holding Times

tween 0.50 and 1.25. This is a favorable result from a practical standpoint, because it suggests that optimization-based holding strategies lead to improved performance even when human factors lead to small errors in applied holding times. However, passenger cost increases significantly for adjustment factors of 1.50 and greater, suggesting that, within the range of adjustment factors considered, excessive holding is more problematic than insufficient holding. Holding for less than what the optimization model suggests allows for errors to be corrected in the future, i.e. the optimization model can suggest even greater holding times to prevent bunching when it is solved again, while excessive holding irreversibly delays passengers. In this case with high crowding, excessive holding leads to full vehicles, many passengers left behind, and hence longer waiting times, which are reflected in the mean passenger costs shown in Figure 8. Although it is not shown in the figure, the standard deviation of mean passenger cost across replications also increases sharply for adjustment factors of 1.50 and greater, suggesting that not only does performance worsen on average, it also becomes more unpredictable.

While the transit line performs better with the OD control strategy for adjustment factors between 0.50 and 1.25 , performance worsens more with the OD strategy than with the OS strategy for adjustment factors greater than 1.25. Since the OD optimization model works with more information, it can lead to a more specific holding strategy. For example, it may allow a bunch to form in order to deal with a temporary capacity shortage in a specific section of the line, and later hold vehicles for longer than usual at the terminal in order to break the bunch formed earlier. If vehicles are held there for longer than is necessary, frequency and capacity decrease, potentially leading to capacity problems once more. 


\subsection{Computation Time}

In the context of real-time control, it is important to consider not only the effectiveness of control but also the computational effort required to generate control policies. This is because in real applications decisions must be made quickly and provided to vehicles soon after their arrival at control points. Simulations were run on a computer having an Intel Core i7-3930K processor running at $3.20 \mathrm{GHz}$. Mean computation times were 0.77 seconds with OS and $1.08 \mathrm{sec}-$ onds with OD. The $90^{\text {th }}$ percentile computation time for the OD strategy was 1.83 seconds, and the maximum was 3.42 seconds. Therefore, it is feasible to employ the OD strategy in real-time applications.

\section{Concluding Remarks}

The mathematical model formulated in this research captures dynamic running times and demand. A holding strategy based on this model (OD) was tested in a simulation environment under a variety of cases having different dynamics, and its performance was compared to that of three other control strategies, including a similar optimization-based strategy that assumes the equivalent static running times and demand (OS). The principal performance measure used for evaluating strategies was mean passenger cost, which reflects passenger trip durations weighting time spent waiting at stops twice as much as time spent in the vehicle. Excess waiting time, headways, vehicle loads, and crowding at stops were also considered. A summary of findings is listed below:

1. The proposed control strategy based on optimization with dynamic inputs (OD) outperforms the OS strategy in cases where, due to running time or demand dynamics, the system becomes overcrowded and passengers are left behind by (full) vehicles. In cases having dynamic running times but not significant overcrowding, the proposed dynamic strategy OD modestly outperforms the OS strategy. Dynamics in demand do not provide an opportunity for the OD strategy to improve performance more than the OS strategy, unless the dynamics lead to significant overcrowding.

2. Optimization-based control strategies lead to similar or better performance than the even headway strategy $(\mathrm{EH})$, and the EH strategy outperforms the (fixed) target headway strategy $(\mathrm{TH})$.

3. Holding strategies improve performance through a combination of running more frequent service due to more efficient use of the fleet and regulating headways. The performance improvement going from $\mathrm{TH}$ to $\mathrm{EH}$ is at least in part due to running more frequent service. However, headway regulation is the principal mechanism by which performance improves going from $\mathrm{EH}$ to OS and from OS to OD.

4. When dynamics are present, it is possible for a holding strategy to improve performance even if its holding policies lead to increased headway variability (and similar mean headways), especially if the increase in headway variability is a byproduct of more efficient use of resources and the 
optimal holding policy is different from merely balancing headways. This is relevant in cases of overcrowded operations.

5. The degree to which the OD strategy outperforms the OS strategy is not sensitive to running time variability. Most of the benefit comes from the OD strategy's ability to generate preemptive holding policies in light of forecast dynamics.

6. A large part of the performance improvement going from OS to OD is due to more accurate estimates of the current state, while the remainder comes from modeling running times and demand dynamically when forecasting future system states under varying holding policy scenarios.

7. Performance under both the OS and the OD strategies is robust to the application of holding times slightly different from those suggested by the optimization model, but declines sharply when the applied holding times greatly exceed those suggested by the optimization model, more so under the OD strategy than under the OS strategy.

8. Computation times with the OD strategy are suitable for real-time application.

The findings presented in this paper are specific to the hypothetical transit system studied using simulation, although they might also hold for many real high-frequency transit services. Future research could explore the effectiveness of the proposed dynamic holding strategy in a real transit system. In real applications it may be undesirable to delay vehicles whose drivers are expected to arrive late at their scheduled relief point. The incorporation of crew constraints and their effect on control effectiveness could be explored. The effect of intentional or benign disregard of holding instructions by drivers could be investigated. In this research, holding optimization uses error-free information about future mean running times and demand, for both the OS and the OD strategies. Information errors, in particular of vehicle locations, loads, running times, and demand, might lead to different results, so their effect on holding policies and performance should be investigated. Control strategies that capture the stochastic aspects of transit system performance could be developed and tested, and the relative importance of capturing stochasticity vs. capturing dynamics could be investigated.

\section{Acknowledgements}

This research is funded in part by the National Science Foundation Graduate Research Fellowship Program under Grant No. 1122374 and in part by Transport for London. This research also benefited from the support of the Bus Rapid Transit Centre of Excellence, funded by the Volvo Research and Educational Foundations (VREF), and MIT's MISTI-Chile program. Juan Carlos Muñoz, Felipe Delgado, and Ricardo Giesen from Pontificia Universidad Católica de Chile gave invaluable feedback in the early stages of this research. 


\section{References}

Abkowitz, M., Lepofsky, M., 1990. Implementing Headway-Based Reliability Control on Transit Routes. Journal of Transportation Engineering 116 (1), 49-63.

Adamski, A., Turnau, A., 1998. Simulation support tool for real-time dispatching control in public transport. Transportation Research Part A: Policy and Practice 32 (2), 73-87.

Barnett, A., 1974. On Controlling Randomness in Transit Operations. Transportation Science 8 (2), 102-116.

Bartholdi, J. J., Eisenstein, D. D., 2012. A self-coördinating bus route to resist bus bunching. Transportation Research Part B: Methodological 46 (4), 481491.

Berrebi, S. J., Watkins, K. E., Laval, J. A., 2015. A real-time bus dispatching policy to minimize passenger wait on a high frequency route. Transportation Research Part B: Methodological, - .

Cats, O., Larijani, A. N., Koutsopoulos, H. N., Burghout, W., 2011. Impacts of Holding Control Strategies on Transit Performance: Bus Simulation Model Analysis. Transportation Research Record: Journal of the Transportation Research Board 2216 (1), 51-58.

Chandrasekar, P., Cheu, R. L., Chin, H. C., 2002. Simulation Evaluation of Route-Based Control of Bus Operations. Journal of Transportation Engineering 128 (6).

Chen, Q., Adida, E., Lin, J., 2013. Implementation of an iterative headwaybased bus holding strategy with real-time information. Public Transport 4 (3), $165-186$.

Daganzo, C. F., 2009. A headway-based approach to eliminate bus bunching: Systematic analysis and comparisons. Transportation Research Part B: Methodological 43 (10), 913-921.

Daganzo, C. F., Pilachowski, J., 2011. Reducing Bunching with Bus-to-Bus Cooperation. Transportation Research Part B: Methodological 45 (1), 267277 .

Delgado, F., Muñoz, J. C., Giesen, R., 2012. How much can holding and/or limiting boarding improve transit performance? Transportation Research Part B: Methodological 46 (9), 1202-1217.

Delgado, F., Muñoz, J. C., Giesen, R., Cipriano, A., 2009. Real-time control of buses in a transit corridor based on vehicle holding and boarding limits. Transportation Research Record: Journal of the Transportation Research Board 2090 (1), 59-67. 
Dessouky, M., Hall, R., Zhang, L., Singh, A., 2003. Real-time control of buses for schedule coordination at a terminal. Transportation Research Part A: Policy and Practice 37 (2), 145-164.

Eberlein, X. J., Wilson, N. H. M., Bernstein, D., 2001. The Holding Problem with Real-Time Information Available. Transportation Science 35 (1), 1-18.

Gordon, J. B., Koutsopoulos, H. N., Wilson, N. H., Attanucci, J. P., 2013. Automated Inference of Linked Transit Journeys in London Using Fare-Transaction and Vehicle Location Data. Transportation Research Record: Journal of the Transportation Research Board 2343 (1), 17-24.

Hernández, D., Muñoz, J. C., Giesen, R., Delgado, F., 2015. Analysis of realtime control strategies in a corridor with multiple bus services. Transportation Research Part B: Methodological 78, 83-105.

Ibarra-Rojas, O., Delgado, F., Giesen, R., Muñoz, J., 2015. Planning, operation, and control of bus transport systems: A literature review. Transportation Research Part B: Methodological 77, 38-75.

McCord, M. R., Mishalani, R. G., Goel, P., Strohl, B., 2010. Iterative proportional fitting procedure to determine bus route passenger origin-destination flows. Transportation Research Record: Journal of the Transportation Research Board 2145 (1), 59-65.

Muñoz, J. C., Cortés, C. E., Giesen, R., Sáez, D., Delgado, F., Valencia, F., Cipriano, A., 2013. Comparison of dynamic control strategies for transit operations. Transportation Research Part C: Emerging Technologies 28 (0), 101113.

Newell, G. F., 1974. Control of Pairing of Vehicles on a Public Transportation Route, Two Vehicles, One Control Point. Transportation Science 8 (3), 248264.

Osuna, E. E., Newell, G. F., 1972. Control Strategies for an Idealized Public Transportation System. Transportation Science 6 (1), 52-72.

Powell, M. J., 2009. The BOBYQA algorithm for bound constrained optimization without derivatives. Cambridge NA Report NA2009/06, University of Cambridge, Cambridge.

Puong, A., Wilson, N. H., 2008. A train holding model for urban rail transit systems. In: Hickman, M., Mirchandani, P., Voß, S. (Eds.), Computer-aided Systems in Public Transport. Springer, pp. 319-337.

Rossetti, M. D., Turitto, T., 1998. Comparing static and dynamic threshold based control strategies. Transportation Research Part A: Policy and Practice 32 (8), 607-620. 
Sáez, D., Cortés, C. E., Milla, F., Núñez, A., Tirachini, A., Riquelme, M., 2012. Hybrid predictive control strategy for a public transport system with uncertain demand. Transportmetrica 8 (1), 61-86.

Sánchez-Martínez, G. E., 2012. Running Time Variability and Resource Allocation: A Data-Driven Analysis of High-Frequency Bus Operations. Master's thesis, Massachusetts Institute of Technology.

Shen, S., Wilson, N. H. M., 2001. An Optimal Integrated Real-Time Disruption Control Model for Rail Transit Systems. Computer-Aided Scheduling of Public Transport, 335-363.

Sun, A., Hickman, M., 2008. The holding problem at multiple holding stations. In: Hickman, M., Mirchandani, P., Voß, S. (Eds.), Computer-aided systems in public transport. Springer, pp. 339-359.

Turnquist, M. A., Blume, S. W., 1980. Evaluating potential effectiveness of headway control strategies for transit systems. Transportation Research Record: Journal of the Transportation Research Board (746), 25-29.

Xuan, Y., Argote, J., Daganzo, C. F., 2011. Dynamic Bus Holding Strategies for Schedule Reliability: Optimal Linear Control and Performance Analysis. Transportation Research Part B: Methodological 45 (10), 1831-1845.

Yu, B., Yang, Z., 2009. A dynamic holding strategy in public transit systems with real-time information. Applied Intelligence 31 (1), 69-80.

Zhao, J., Bukkapatnam, S., Dessouky, M. M., 2003. Distributed architecture for real-time coordination of bus holding in transit networks. IEEE Transactions on Intelligent Transportation Systems 4 (1), 43-51.

\section{Appendix A. Mathematical Notation}

$\begin{array}{ll}V & \text { set of vehicles } \\ n_{V} & \text { number of vehicles, i.e. the size of set } V \\ S & \text { set of stops } \\ n_{S} & \text { number of stops, i.e. the size of set } S \\ t & \text { time } \\ t_{0} & \text { current time } \\ e_{v} & \text { last stop vehicle } v \text { departed from before } t_{0} \\ g_{s} & \text { next vehicle that will depart stop } s \text { after } t_{0} \\ a_{v, s} & \text { unconstrained arrival time of vehicle } v \text { at stop } s \\ d_{v, s} & \text { unconstrained departure time of vehicle } v \text { from stop } s \\ \bar{a}_{v, s} & \text { constrained arrival time of vehicle } v \text { at stop } s \\ \bar{d}_{v, s} & \text { constrained departure time of vehicle } v \text { from stop } s \\ a_{v, s}^{\prime} & \text { arrival time of vehicle } v \text { at stop } s \text { in the previous cycle, i.e. before } \\ & t_{0}\end{array}$




\begin{tabular}{|c|c|}
\hline$d_{v, s}^{\prime}$ & $\begin{array}{l}\text { departure time of vehicle } v \text { from stop } s \text { in the previous cycle, i.e. be- } \\
\text { fore } t_{0}\end{array}$ \\
\hline$r_{s}(t)$ & $\begin{array}{l}\text { unconstrained running time from stop } s \text { to the next stop when } \\
\text { departing } s \text { at time } t\end{array}$ \\
\hline $\bar{r}_{v, s}$ & $\begin{array}{l}\text { xtra running time of vehicle } v \text { from stop } s \text { to the next stop due to } \\
\text { locking, i.e. when the overtaking constraint is binding }\end{array}$ \\
\hline $\bar{r}_{v}^{\prime}$ & $\begin{array}{l}\text { extra running time of vehicle } v \text { from the stop it last departed before } \\
t_{0}\left(e_{v}\right) \text { to its next stop }\left(e_{v}+1\right) \text { due to blocking }\end{array}$ \\
\hline$\delta_{v, s}$ & well time of vehicle $v$ at stop $s$ \\
\hline$h_{v, s}$ & holding time of vehicle $v$ at stop $s$, the decision variable \\
\hline $\bar{h}_{v, s}$ & $\begin{array}{l}\text { extra holding time of vehicle } v \text { at stop } s \text { due to blocking, i.e. when } \\
\text { the overtaking constraint is binding }\end{array}$ \\
\hline$\tau_{b}$ & boarding time per passenger \\
\hline$\tau_{a}$ & alighting time per passenger \\
\hline$\beta_{v, s_{b}, s_{a}}$ & $\begin{array}{l}\text { number of passengers waiting to board vehicle } v \text { at stop } s_{b} \text { and } \\
\text { alight at stop } s_{a}\end{array}$ \\
\hline & number of passengers waiting to board vehicle $v$ at stop $s$ \\
\hline $\bar{\beta}_{v, s_{b}, s_{a}}$ & $\begin{array}{l}\text { number of passengers who board vehicle } v \text { at stop } s_{b} \text { and alight at } \\
\text { stop } s_{a}\end{array}$ \\
\hline $\bar{\beta}_{v, s}$ & number of passengers who board vehicle $v$ at stop $s$ \\
\hline$\alpha_{v, s}$ & number of passengers who alight vehicle $v$ at stop $s$ \\
\hline$l_{v, s}$ & load of vehicle $v$ arriving at stop $s$ \\
\hline$l_{v, s_{a}}^{\prime}$ & number of passengers in vehicle $v$ at time $t_{0}$ who alight at stop $s_{a}$ \\
\hline$\pi_{v, s_{b}, s_{a}}$ & $\begin{array}{l}\text { mber of passengers prevented from boarding vehicle } v \text { at stop } s_{b} \\
\text { o alight at stop } s_{a}\end{array}$ \\
\hline$\pi_{v, s}$ & number of passengers prevented from boarding vehicle $v$ at stop $s$ \\
\hline$k$ & us capacity \\
\hline$\lambda_{s_{b}, s_{a}}(t)$ & arrival rate of passengers who board at stop $s_{b}$ and alight at stop \\
\hline$\iota_{s_{b}, s_{a}}^{\prime}$ & $\begin{array}{l}s_{a}, \text { as a function of time } t \\
\text { number of passengers already waiting at stop } s_{b} \text { at time } t_{0} \text { destined } \\
\text { for stop } s_{a}\end{array}$ \\
\hline$W_{V}$ & total in-vehicle delay time of passengers due to holding \\
\hline$W_{S}$ & total waiting time of passengers o \\
\hline$\theta_{S}$ & relative weight of holding time at stops \\
\hline$W_{S_{0}}$ & waiting time of passengers who arrive before $t_{0}$ \\
\hline$W_{S_{1}}$ & $\begin{array}{l}\text { waiting time of passengers who arrive after } t_{0} \text { but before the first } \\
\text { vehicle departs from that stop in the model horizon }\end{array}$ \\
\hline$W_{S_{2}}$ & $\begin{array}{l}\text { waiting time of passengers who arrive after the first vehicle departs } \\
\text { from that stop in the model horizon, excluding extra waiting time } \\
\text { when left behind }\end{array}$ \\
\hline$W_{S_{3}}$ & extra waiting time of passengers left behind \\
\hline & tal boardings over the model $\mathrm{h}$ \\
\hline
\end{tabular}

